\title{
Profiling and engineering of microRNAs for enhancing recombinant protein productivity in Chinese hamster ovary cells
}

\author{
Wan Ping Loh", Bernard Loo1, Lihan Zhou², Peiqing Zhang ${ }^{1}$, Dong Yup Lee ${ }^{1,2}$, Yuan Sheng Yang ${ }^{1}$, \\ Kong Peng Lam ${ }^{1}$
}

From 23rd European Society for Animal Cell Technology (ESACT) Meeting: Better Cells for Better Health Lille, France. 23-26 June 2013

\section{Background}

Chinese hamster ovary $(\mathrm{CHO})$ cells have become dominant host cells in the biopharmaceutical industry due to their capacity for proper protein folding, assembly and posttranslational modifications. However, low specific productivity (qp) places limitations on yields obtained from mammalian host cells. MicroRNAs (miRNAs), a novel class of short, non-coding RNAs which negatively regulate target gene expression at post-transcriptional levels, have emerged as promising targets for engineering of $\mathrm{CHO}$ cell factories to enhance recombinant protein production. While engineering of miRNAs for enhanced cell growth and delayed cell death have been reported, miRNA targets which can enhance qp have not been identified to date.

\section{Materials and methods}

To understand the role of miRNAs in conferring high qp phenotype in $\mathrm{CHO}$ cells, we carried out high throughput sequencing of 4 in-house generated IgGexpressing $\mathrm{CHO}$ sub-clones of varying qps. Reads were mapped to miRBase and 22 miRNAs were found to be differentially expressed between the high and low producers. These miRNAs were stably transfected into an IgG-expressing sub-clone to assess their effects on growth, titer, qp and product quality attributes.

\section{Results}

Over-expression of miRs-17, 19b, 20a and 92a individually and in combination resulted in $13-27 \%$ increases in titer and $14-24 \%$ increases in qp in stably transfected pools. No significant alterations in proliferation rates

${ }^{1}$ Bioprocessing Technology Institute, 20 Biopolis Way, \#06-01 Centros, Singapore 138668

Full list of author information is available at the end of the article were observed. 20-45 single cell clones were randomly selected from each of the 5 transfected pools for characterization. Statistical analyses showed significant differences in titer/qp between the high- and low-miRNA expressing single cell clones. The highest producing single cell clones exhibited $\sim 100 \%$ increases in titer and qp compared to non-transfected cells. A correlation was found between increased miR-19b levels (>1.3-fold) and enhanced qp and titer. Over-expression of miR-19b does not appear to impact IgG aggregation significantly.

\section{Conclusions}

To our knowledge, this is the first report of enhancement of recombinant protein productivity by stable miRNA over-expression. The genes and cellular pathways targeted by these miRNAs specific to enhancing protein productivity are under investigation and will be reported.

\section{Acknowledgements \\ This work was supported by the Biomedical Research Council/Science and Engineering Research Council of A*STAR (Agency for Science, Technology and Research), Singapore. The authors would like to thank Faraaz Noor Khan Yusufi, Ju Xin Chin for their assistance in processing of next-generation sequencing data, and Corrine Wan, Gavin Teo, Daniel Chew, Lyn Chiin Sim, Ce Huang Poo and Kong Meng Hoi for their technical assistance in IgG purification, aggregation and glycosylation analyses.}

\section{Authors' details \\ 'Bioprocessing Technology Institute, 20 Biopolis Way, \#06-01 Centros, Singapore $138668 .{ }^{2}$ Department of Biochemistry, National University of Singapore, 8 Medical Drive 4, Blk MD7 \#05-04, Singapore 117597.}

Published: 4 December 2013

doi:10.1186/1753-6561-7-S6-P102

Cite this article as: Loh et al:: Profiling and engineering of microRNAs for enhancing recombinant protein productivity in Chinese hamster ovary cells. BMC Proceedings 2013 7(Suppl 6):P102. 